\title{
miR-302e Suppresses Glioma Progression by Targeting VEGFA
}

This article was published in the following Dove Press journal:

Cancer Management and Research

\section{Yunpeng Xie' \\ Xin Liu $^{2}$ \\ Tiemin $\mathrm{Hu}^{\prime}$ \\ Weixing Wang'}

'Department of Neurosurgery, Chengde Medical College Affiliated Hospital, Chengde, Hebei Province, People's Republic of China; ${ }^{2}$ Department of Oncology, Chengde Medical College Affiliated Hospital, Chengde, Hebei

Province, People's Republic of China
Background: MiRNA can be involved in regulating tur genesis and evelopment by regulating the expression of specific genes and regulat 8 corre anding sig ling pathways. In this study, we explored the function and mechar ms of miR-30 in oma progression. Methods: Experimental methods include the fo wing: $r$-time quar atative PCR, Western Blot Analysis, CCK8 assay and detection 0 apop

Results: MiR-302e was down-regulate cancer tiss an expression of miR-302e was negatively correlat with tumor gra which indicated poor prognosis in glioma patients. Followed functional analys showed overexpression of miR-302e inhibited proliferation, migration anvasion but $p_{\text {a }}$ oted apoptosis of glioma cells, while silencing miR-302e show the opposita effects. Mechanistic studies have shown that VEGFA was a directed to et of miR-3 e. Forced expression of VEGFA removed the inhibiting impact of miR-30, on gliom development. In vivo tumorigenesis experiments showed that mil appressan development by targeting VEGFA.

Conclusion: Pre nt stur whasized miR-302e suppressed glioma development by targeting VICGFA, wh hight be a valuable target for glioma treatment.

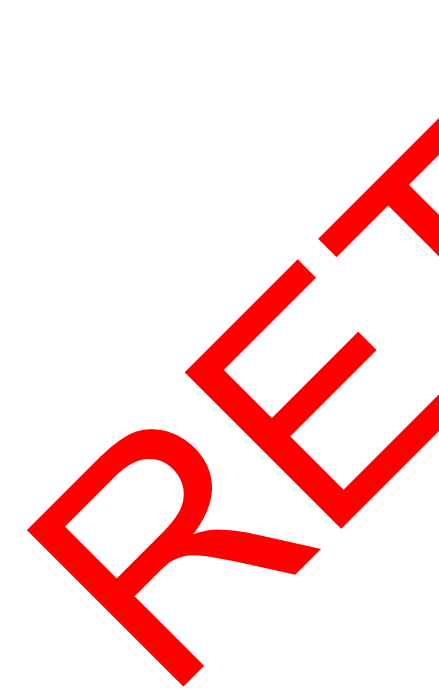

Correspondence: Weixing Wang Department of Neurosurgery, Chengde Medical College Affiliated Hospital, No. 36, Nanyingzi Avenue, Chengde, Hebei Province, People's Republic of China Email yinyuti00377360@I63.com

\section{Key ords: loma, R-302e, proliferation, migration, VEGFA}

\section{Intro yction}

Clioma is a common primary central nervous system tumor. ${ }^{1}$ The biological ch. cteristics of glioma, such as abundant blood vessels, invasive growth, and high recurrence rate, make the prognosis of glioma patients poor, which is also a significant problem that affects long-term neurosurgery. The higher the pathological grade of glioma, the stronger its invasion, the more blurred the boundary between tumor tissue and healthy brain tissue, significantly increasing the difficulty of surgical resection and chemoradiotherapy resistance. ${ }^{2}$ Although in recent years, glioma surgery, chemoradiotherapy and other treatment technology have made significant progress. However, the five-year survival rate for glioma patients is still less than $3 \% .^{3}$ It is found that glioma belongs to a polygenic lesion in nature, and its occurrence and development are regulated by multiple genes. ${ }^{4}$ Therefore, it has become a novel breakthrough point in glioma treatment to study the relationship between related factors at gene regulation level and to seek new molecular gene targets.

MiRNAs are a class of endogenous non-coding small RNA with a length of 18-24 nucleotides. ${ }^{5}$ MiRNAs are ubiquitous in viruses and eukaryotes, and their sequences are highly conserved. ${ }^{6}$ According to statistics, up to $50 \%$ of protein- 
coding genes in all humans are regulated by miRNAs. ${ }^{7}$ Each miRNA regulates up to hundreds of different RNAs and is associated with tissue specificity and disease conditions. In gliomas, miRNA expression can be altered through a variety of mechanisms, including chromosomal changes, epigenetic defects, and mutations. ${ }^{8}$ Because of the unique biological properties of miRNAs and the importance of their roles, they can be used as diagnostic biomarkers. In malignant tumors, miRNA s can be used to assess trends in cancer invasion, metastasis, or drug resistance as prognostic indicators. ${ }^{9-11}$ Therefore, miRNA can be involved in regulating tumor genesis and development by regulating the expression of certain genes and regulating corresponding signaling pathways, which provides a new way for the diagnosis, treatment and prognosis of tumors.

Cancerous cells are abnormally enhanced in proliferation, invasion, metastasis and angiogenesis, which changes the standard expression regulation mechanism of cells. ${ }^{12,13}$ The genesis and development of the tumor is a multi-stage complex process, which usually includes unlimited proliferation, decreased apoptosis and angiogenesis. Tumor cells have a strong ability to survive in the body, but the living body itself cannot carry out adequate immune clearan Early researchers tried to find miRNAs with differentia expression in tumor samples and normal sampl as to find new target indicators for tumor treat cnt. ${ }^{14,1}$ The microarray results showed that 30 miRNA vere tially expressed, some of which inve din the formation and metastasis of glio

MiR-302e is a broadly conservativ viRNA, ${ }^{17}$ which has been reported to play in essential fû ion in colorectal cancer, ovarian ancer $\mathrm{d}$ non-small cell lung cancer. ${ }^{18-20}$ And it $\mathrm{wa}_{\mathrm{a}}$ sh $\mathrm{n}$ that niR-302e targeted VEGFA in ova now cr, the role of miR302e in glig a still mains stinct. In this study, we focused on fy miR-302e and the possible mechanisms in oma, which might contribute to a potential drug target for joma treatment.

\section{Methods}

\section{Clinical Samples}

The surgical specimens of 30 glioma patients from our hospital were collected, which were used for follow-up experimental detection. The experiment was permitted by the Ethics Committee of Chengde Medical College Affiliated Hospital, and the patients signed informed consent.

\section{Cell Culture}

Cell lines (normal human astrocytes, SHG44, U251, U87 and SHG139) were purchased from CHI Scientific, Inc (Jiangsu, China). The cells were cultured with complete medium including 89\% 1640 and 10\% FBS, both were purchased from Biological Industries (Beit-Haemek, Israel), and maintained in incubator with $37^{\circ} \mathrm{C}$ and $5 \%$ of $\mathrm{CO}_{2}$ saturated humidity.

\section{Cell Transfection and Treatment}

The cells were plated until the cell dep $80 \%$ confluency of dishes to transfect. Ago R-302e, m 302e inhibitor, VEGFA plasmid or NC yere structed by fenechem (Shanghai, China). The RN or plasmt are tra ected with Lipofectamine 2000 (In ogen, $C$ Asbad,

\section{qRT-PCR}

RNA extra on is perforn d using trizol reagent. NanoDron 8000 (1) rmo Scientific, Waltham, MA, USA was used to detecm the concentration and purity of $\mathrm{RN}$. The singl stranded cDNAs were synthesized from $1 \mu$ of RNA. $T$ e expression of mRNAs and miRNAs were q. tifie oy RT-PCR with SYBR Green I (Thermo - Scientific, Inc). Primer list: miR-302e (F: 5'CGIICCAGTGCAGGGTCCGAGGTATTCGCACTGGATACGACAAGCAT -3', R: 5'-ATACTCGTAAGT CTTCCATGCTT -3'), VEGFA (F: 5'- TATTCAGCGG ACTCACCAGC -3', R: 5'- AACCAACCTCCTCA AACCGT -3'), U6 (F: 5'-TCGCCCTTGGCA CAGCA3', R: 5'-CGAACCATTCAAGTGTTGCT-3'), GADPH (F: 5'- CTCCTGCACCACCAACTGCT -3', R: 5'-GGG CCATCCACAGTCTTCTG -3').

\section{Protein Isolation and Western Blot}

After RIPA cleavage, we extracted total protein and measured with BCA method. After quantitative denaturation, protein electrophoresis membrane transfer and blocked. The first incubation and second incubation were carried out according to the operation steps. The expression of the protein was expressed by the gray value. Primary antibodies list: VEGFA (66,828-1-Ig, Proteintech), Bcl2 (ab182858, Abcam), Bax (ab32503, Abcam), Caspase3 (ab13847, Abcam), GADPH (60,004-1-Ig, Proteintech).

\section{CCK8 Assay}

Cells were plated in 96-well plates and we used CCK8 assay to detect the cell viability. CCK 8 (10 nmol/L; 
Beyotime Biotechnology, China) was added after curcumin treatment and incubated at $37^{\circ} \mathrm{C}$. We measured the absorbance of $450 \mathrm{~nm}$ at 24, 48 and $72 \mathrm{~h}$.

\section{Detection of Apoptosis}

The Annexin V-FITC/PI apoptosis kit was purchased from Solebao Company (Beijing, China), and an appropriate amount of logarithmic growth phase cells were washed twice with pre-cooled PBS. The MKN-45 cells were suspended with $500 \mathrm{ul}$ of bound buffer, mixed with $5 \mu \mathrm{l}$ of annexin V-FITC and PI, respectively, and placed at $25^{\circ} \mathrm{C}$ for $15 \mathrm{~min}$.

\section{Animals}

Animal experiments were permitted by the Animal Protection and Ethics Committee of Chengde Medical College Affiliated Hospital. BALB/c nude mice (6-8 weeks) were purchased from Beijing Weitong Lihua Experimental Animal Technology Co., Ltd. (Beijing, China). For the experiment of Xenograft, SHG44 cell cells $\left(5 \times 10^{6}\right)$ were suspended in $200 \mu \mathrm{L}$ normal saline and subcutaneously injected or through tail vein. Tumor volume $\left(\mathrm{mm}^{3}\right): \mathrm{V}\left(\mathrm{Mm}^{3}\right)=\mathrm{S} 2\left(\mathrm{Mm}^{2}\right) \times \mathrm{L}$ $(\mathrm{Mm}) / 2$. All animal experiments were perform compliance with institutional guidelines and had approved by SJTUSM Institutional and Use Committee (Protocol $r$ gistry) Numbe A-2018-014).

Table I Clinical Characterist of $G$ na Patients

\begin{tabular}{|l|l|l|}
\hline Characteristics & N (30) \\
\hline Age (median; min-m & \\
\hline Gender & 19 & 36.67 \\
Male & & 63.33 \\
Femr & 4 & \\
\hline Race & 23 & 13.33 \\
Black & 1 & 76.67 \\
White & 2 & 3.33 \\
Mixed & & 6.67 \\
Other & 12 & \\
\hline WHO grade & 18 & 40.00 \\
I/II & & 60.00 \\
III/IV & 20 & \\
\hline Tumor size (cm) & 10 & 33.33 \\
$<4.5 \mathrm{~cm}$ & & \\
$\geq 4.5 \mathrm{~cm}$ & & \\
\hline
\end{tabular}

\section{Statistical Analysis}

Significant differences were calculated using two-tailed $t$-test through Graphpad 7.0 and SPSS 22.0. The results were presented in form of mean \pm standard deviation (SD). Comparison between the 2 sets of data was performed using Student's $t$-test, and differences between the 3 or more groups of data were compared by one-way ANOVA. All experiments were carried repeatedly for 3 times. $\mathrm{P}<0.05$ signified a statistical significance $(* p<0.05, * * p<0.01)$.

\section{Results}

MiR-302e is Decreas in Gli na Tissues and Associated with $\mathrm{N}$ or Pro losis in Patients

We collected nor and ancer tissu.cs from 30 glioma patients (Tabl a), an croarra was used to determine differentia pression o niP As. And we found miR302e w, a a rential expressed miRNA with a lower level in glioma th es (Figure 1A). Followed qRT-PCR wa showed that mir-302e increased in cancer tissues han norma issues (Figure 1B). We then analyzed the pression o miR-302e in patients with different grades of and found that the miR-302e was negatively rrelated with the tumor grade (Figure 1C). According to the median expression level of miR-302e, the level of miR-302e in glioma tissues was divided into low expression and high expression. Kaplan-Meier curves indicated that 5-year survival rate high expression of miR-302e was significantly lower than low expression patients (Figure 1D). Also, miR-302e was decreased in glioma cell lines (SHG44, U251, U87 and SHG139) comparing with human astrocytes (Figure 1E). The results indicated that miR-302e may play a key role in glioma progression.

\section{MiR-302e Suppresses Proliferation and Promotes Apoptosis of Glioma Cells}

As shown in Figure 1D, SHG44 cells own the lowest expression of miR-302e, and we chose SHG44 to perform the following experiments. We constructed agomiR-302e to force expression of miR-302e in SHG44 cells (Figure 2A). CCK-8 assay manifested that miR-302e inhibited SHG44 cell ability (Figure 2B). Edu staining showed that miR-302e significantly inhibited proliferation of SHG44 cells (Figure 2C). Flow cytometry revealed that miR-302e accelerated glioma cell apoptosis (Figure 2D). In addition, miR-302e remarkably induced Bax and caspase 3 expression, but reduced $\mathrm{Bcl} 2$ level (Figure 2E). 


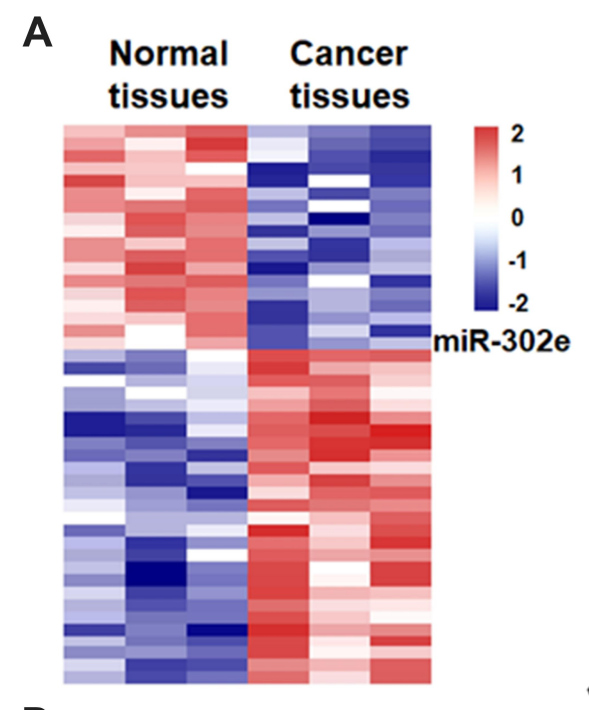

D

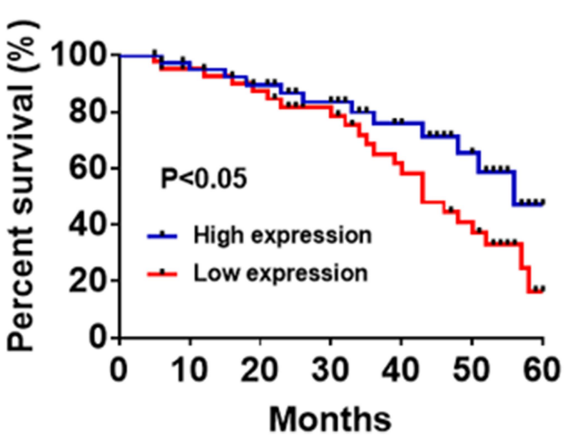

B

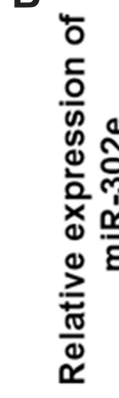

C

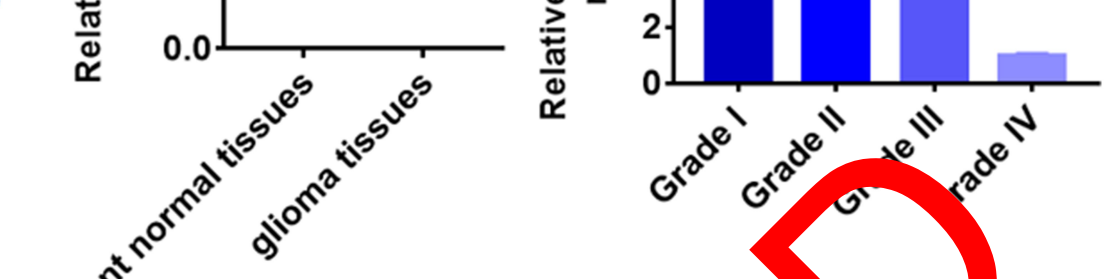

Figure I Expression of miR-302e in glioma tissue and cells. (A) miRNA expression normal tissues $(n=30)$ determined by qRT-PCR $(* * p<0.01)$. (C) Th ion of $m$ III $(n=6)$, and grade IV $(n=4)$ was measured by qRT-PCR, and re relati patients with low or high expression of miR-302e in glioma aes were a ssed by Ka h-Meier survival analysis. (E) $q R T-P C R$ assay analyzed the expression of miR-302e in human astrocytes and glioma cell lines SHG44, U25I, U8, o SHC

\section{MiR-302e Inhibits Mige cio and Invarion of Glioma Cells}

Considering the import ce of prigration an invasion in cancer progression, wherf med yound healing and transwell assay, foun that mir $302 \mathrm{e}$ decreased the wound healin area a $\mathrm{d}$ invas ell numbers (Figure 3A and B).

\section{Inhibition of R-302e Promotes Glioma Progression}

Furthermore, we inhibited miR-302e in U251 cells to evaluate its effect comprehensively. We constructed miR$302 \mathrm{e}$ inhibitor and transfected into U251 cells to suppress miR-302e expression (Figure 4A and B). The data of CCK8 showed that inhibition of miR-302e significantly promoted glioma cell growth. And Edu staining also exhibited a remarkable increase of proliferative cells number in miR-302e transfected cells (Figure 4C). Meanwhile, miR-302e inhibitor decreased the apoptotic cells number in flow cytometry assay (Figure 4D). The wound healing analysis showed that loss of miR-302e elevated glioma migratory ability (Figure 4E). Transwell assay suggested showed that deficiency of miR-302e significantly promoted cell invasion in U251 cells (Figure 4F).

\section{VEGFA is a Direct Target of miR-302e in Glioma Progression}

We used 4 databases to identify the target of miR-302e, and their common intersection showed VEGFA was the most potential target (Figure 5A), which showed that the 3'UTR of VEGFA possessed directed target site for miR302e (Figure 5B). And luciferase activity of WT VEGFA, but not mutant VEGFA, was decreased in the miR-302e group compared with NC group (Figure 5C). Furthermore, 

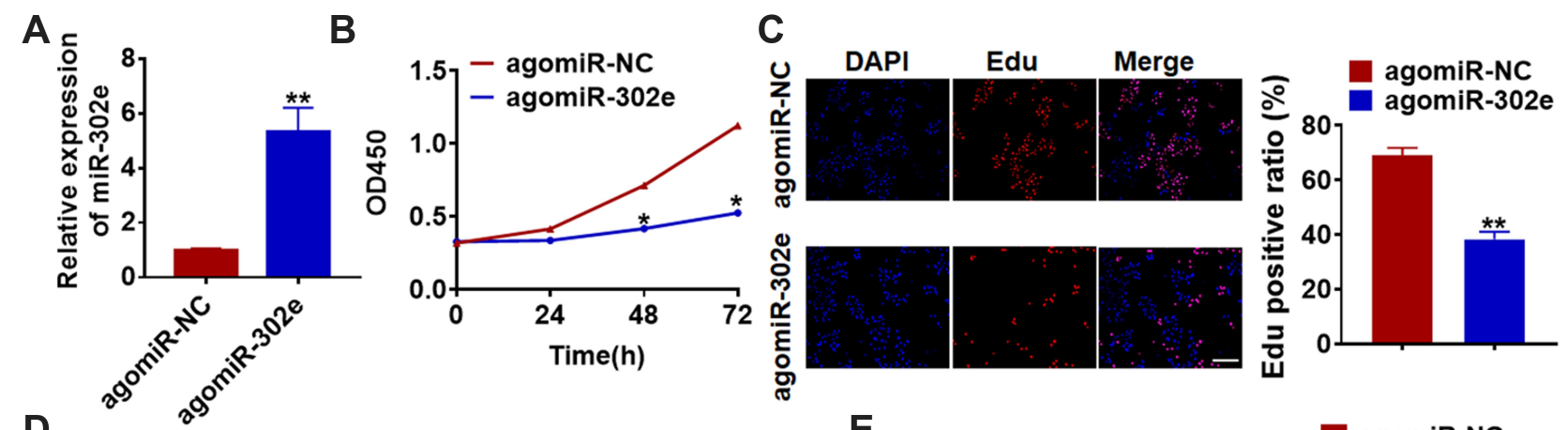

D
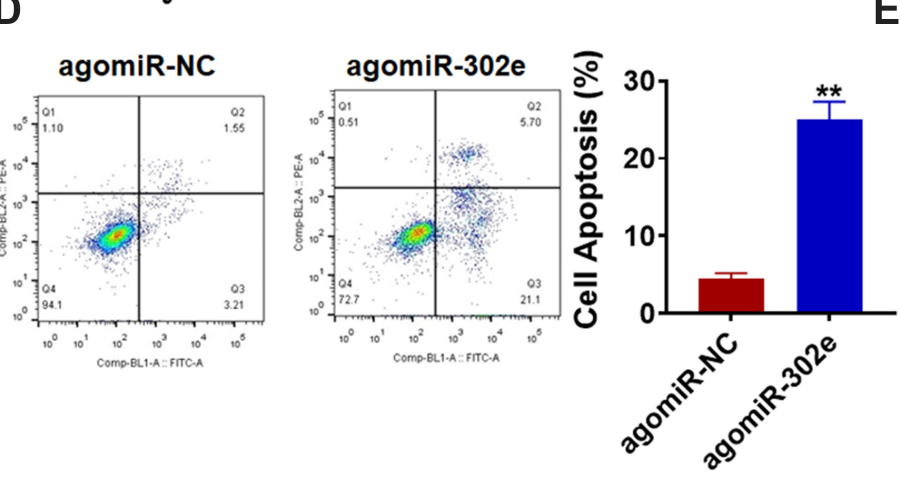

E

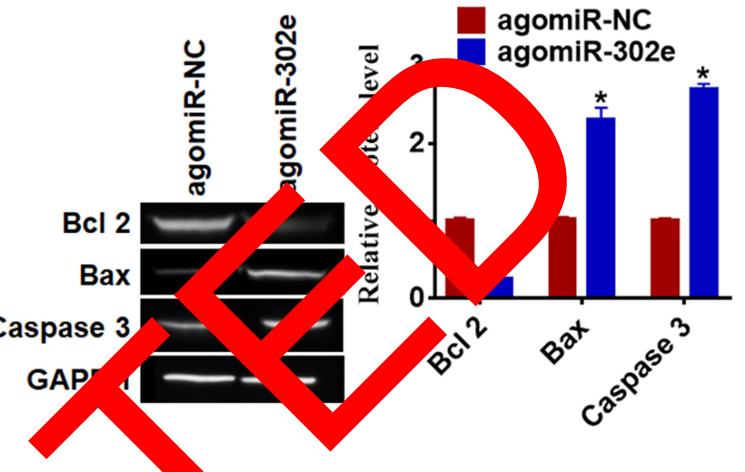

Figure 2 Forced expression of miR-302e inhibits proliferation, but promotes apopto $(* * p<0.0$ I). (B) CKK-8 assay was used to examine the cell growth at $0,24,48$ and 72 r cells. Scale bar, $50 \mu \mathrm{m}(* * p<0.0 \mathrm{I})$. (D) The apoptosis of cells was calculated by flow cy apoptosis related protein $\mathrm{Bcl} 2, \mathrm{Bax}$ and caspase $3\left({ }^{*} p<0.05\right)$. All experiments were car

n SHG44 cells. (A) The expression of miR-302e was determined by qRT-PCR SHG44 cells (*o.05). (C) Edu was used to examine the proliferation of SHG44 netry $(* * p<0.01)$ ) Western blot was performed to detected the expression of repeatedly for times.

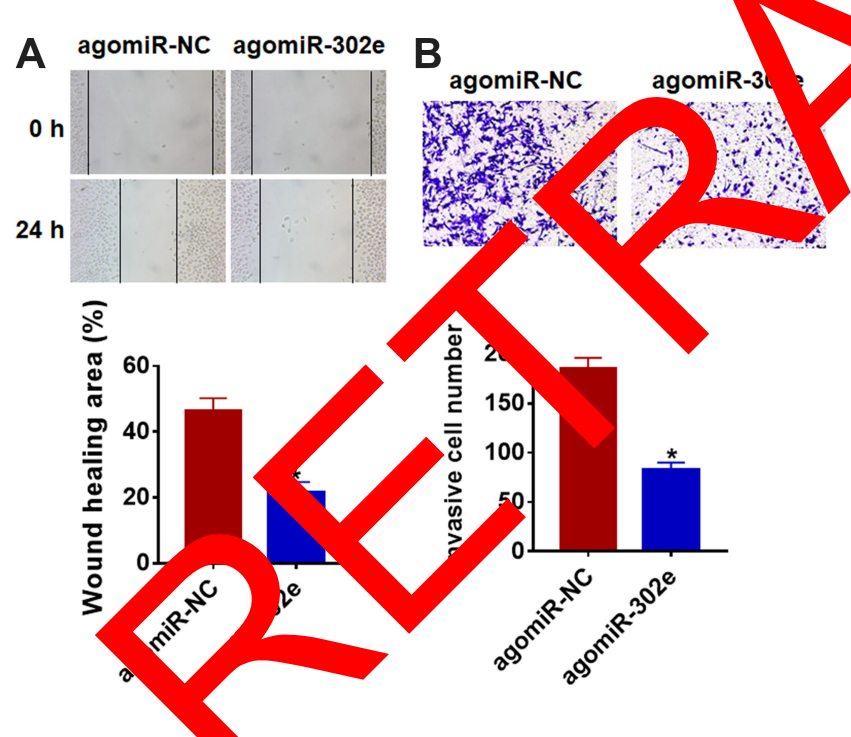

Figure 3 MiR-302e resses migration and invasion in glioma cells. MiR-302e its NC was transfected into SHG44 cells. (A) Wound healing assay was used to detect cell migration $\left({ }^{*} p<0.05\right)$. (B) Transwell assay was performed to check cell invasive ability $\left({ }^{*} p 0.05\right)$. All experiments were carried repeatedly for 3 times.

qRT-PCR analysis showed that agomiR-302e significantly inhibited the expression of VEGFA, while miR-302e inhibitor increased their expression level in SHG44 cells (Figure 5D). In accordance with PCR data, Western blot results exhibited a decrease of VEGFA in miR-302e regulation cells, and an increase in miR-302e downregulation cells (Figure $5 \mathrm{E}$ ). These data suggested that VEGFA might be the targets of miR-302e in glioma modification. What's more, we detected VEGFA expression in clinical glioma tissues and glioma cells. As expected, the protein level of VEGFA was significantly increased in glioma tissue and cells compared with normal tissues and cells (Figure 5F and $\mathrm{G}$ ).

We then forced expression of VEGFA in SHG44 cells (Figure 6A and B). Functionally, VEGFA removed the inhibitory role of miR-302e on glioma progression (Figure 6C-F).

\section{MiR-302e Inhibits in vivo Tumor Growth and Metastasis in the Nude Mice}

For further explore the function of miR-302e on glioma, we set up xenograft nude mice model. Stable miR-302e transfected SHG44 cell lines were constructed, and were injected into nude mice. And we found miR-302e inhibited the growth of glioma (Figure 7A), miR-302e significantly decreased tumors weight (Figure 7B). The expression of miR-302 was increased in the isolated tumors of injected with SHG44 cell stable transfected miR-302e (Figure 7C). 


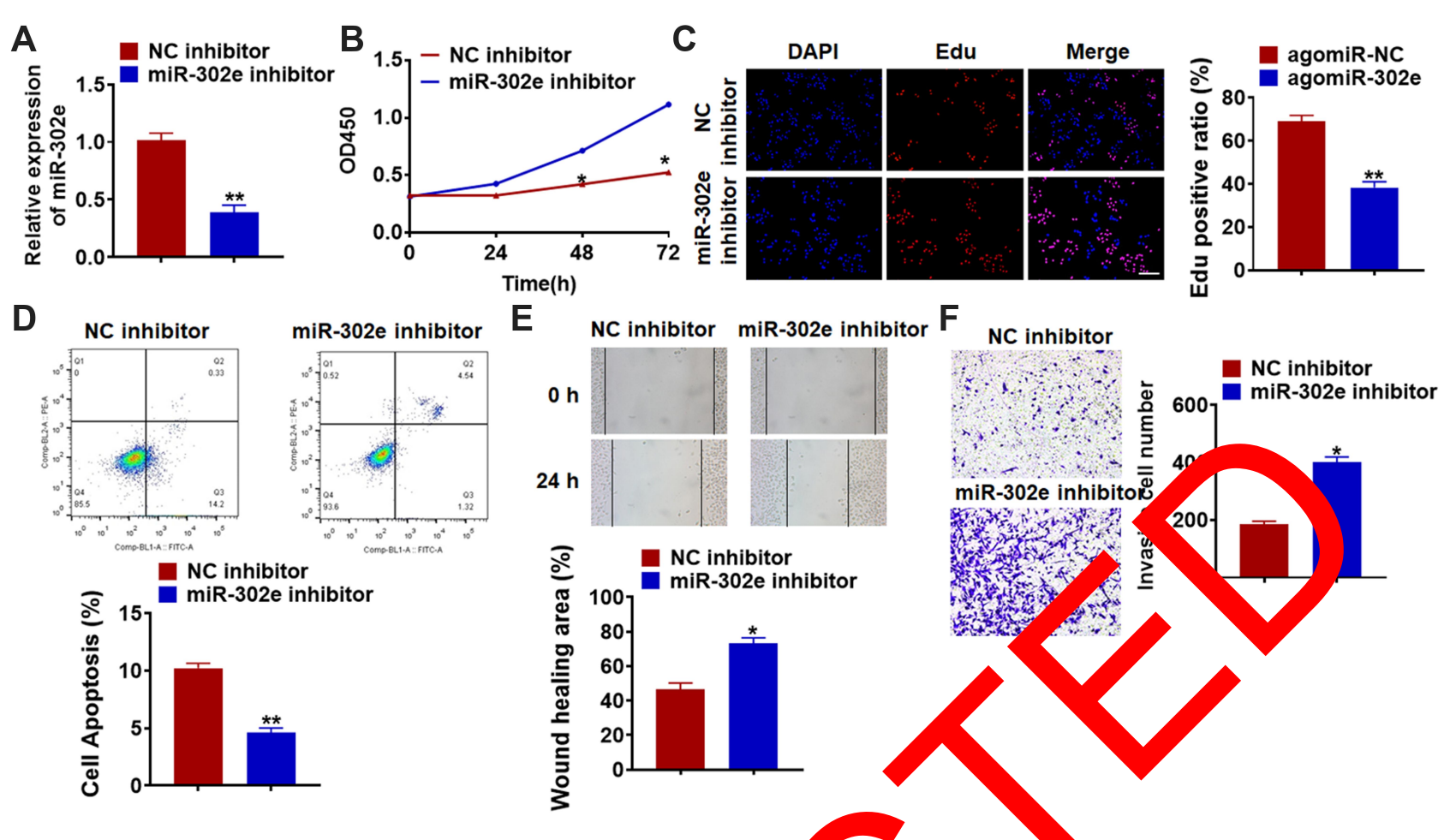

Figure 4 Knockdown of miR-302e promotes proliferation, migration and invasion, but inhibi apoptosis in U25I cells. miR-302e inhibitor or its NC was transfected into U25I cell lines. (A) The expression of miR-302e was determined by qRT-PCR (**p<0.0I). (I CKK-8 assay we sed to examine the cell growth at $0,24,48$ and $72 \mathrm{~h}$ in $\mathrm{U} 25 \mathrm{I}$ cells $\left({ }^{*} p<0.05\right)$. (C) Edu was used to examine the proliferation of U25I cells. Scale bar, $\quad$ um (** $p<0.0 \mathrm{I}$ ). ( The apoptosis of cells was calculated by flow cytometry $(* * p<0.01)$. (E) Wound healing assay was used to detect cell migration $\left({ }^{*} p<0.05\right)$. (F) Transwell ay was perfor d to check cell invasive ability $\left({ }^{*} p<0.05\right)$. All experiments were carried repeatedly for 3 times.

Moreover, miR-302e decreased the mRNA level of coso oompound, polluted air, bad life habit, infection VEGFA in tumor tissues (Figure 7D). And ch, set and other factors concerned. Even with current treatments up another xenograft nude mouse model. $y$ - injecte with at include maximum tumor removal and combined cheSHG44 cell lines firstly. Seven days late. we mectur moradiotherapy, the prognosis for glioma patients is very agomiR-302e and its NC in tumors directly. Interestingly, we got the same alt MiR-302e Juced the tumor volume and ivibited tun growth rate (Figure 7E), miR-302e al significantly dec ased tumors weight (Figure 7F).

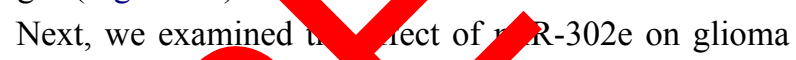
metastasis. Sta $-\mathrm{m}_{\mathrm{m}}-3020$ ans ${ }^{\prime}$ cted SHG44 cell lines were cons acted, 2 d were jected into nude mice through the do nal carn (Figure 8A) and the caudal vein (Figure $8 \mathrm{~b}$, respectively. And we found that miR$302 \mathrm{e}$ significantly hibited pulmonary metastasis of glioma compared with miR-NC. Together, miR-302e could inhibit glioma growth and metastasis, which may improve the prognosis of glioma patients.

\section{Discussion}

Glioma is the deadliest brain tumors. ${ }^{21}$ The etiology that causes this disease is not clear, with tumor origin, genetic factor, biochemical environment, ionizing radiation, poor, with a median survival of only 15 months and only $3 \%$ to $5 \%$ of patients likely to survive longer. ${ }^{22}$ Therefore, it is urgent to explore the pathogenesis of glioma and provide targeted therapy strategies from the molecular perspective.

MiRNAs can be completely or incompletely paired with the 3 'UTR region of target mRNA, ${ }^{23}$ and then inhibit the transcriptional translation of target mRNA, participate in the regulation of biological development and cell growth, and are closely related to the occurrence and development of human tumors. ${ }^{24}$ The discovery of miRNA opens a new window for the study of tumor gene expression regulation. Studies have confirmed the abnormal expression of miRNA in glioma, breast cancer, liver cancer and other malignant tumor stem cells, which affects the proliferation, differentiation, invasion and metastasis of tumor cells by regulating target genes and related signaling pathways. ${ }^{25} \mathrm{MiR}$ $34 \mathrm{c}$ was found to reduce the proliferation rate of breast 


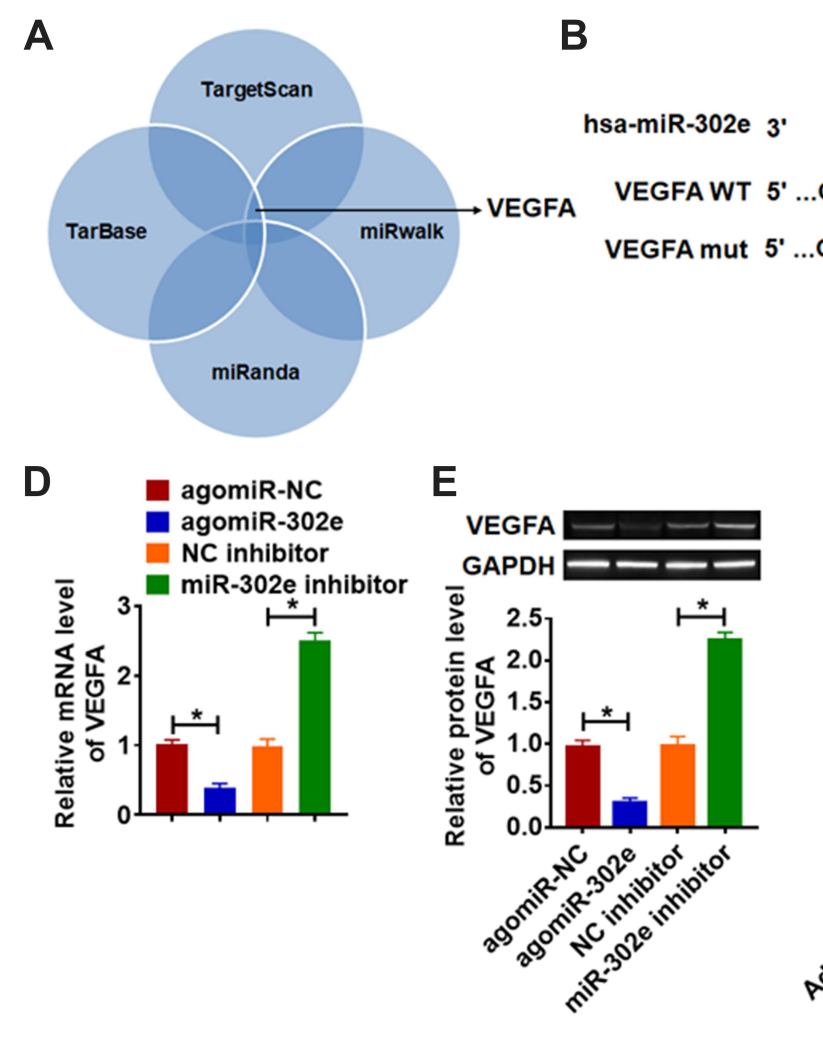

Figure 5 VEGFA is direct targets of miR-302e. (A) 4 databases was used to identify th rrget of miR-30 mutant VEGFA luciferase plasmids were transfected into HEK293 cells with miR-NC or $302 \mathrm{e}$. The ciferase activity was measured by dual-luciferase reporter assay system. miR-302e or miR-302e inhibitor or its NC was transfected into re mRNA level of VEGFA was analyzed by qRT-PCR (* $p<0.05)$. (E) Western blot was performed to detect VEGFA protein expression of $(* p<0$. Thes level of VEGFA in glioma tissues (F) and cells $(\mathbf{G})$ was detected by Western blot $(* * p<0.01)$. All experiments were carried repeatedly for 3 times.

cancer cells. ${ }^{26}$ MiR-105 expression sas sif ificant down-regulated in stem cell cancer 11 lip ical liver cancer tissues, whil Inhibit of miR-105 would promote the prolife a of liver ncer cells and promote tumor formation. Abnormal miRNA expression in specify amors is relate the activation pathway of gene pressig, and miRNA is highly conserved. Therefore, W As can used as high-quality markers to pro te th etic basis for the diagnosis, treatme and ognosis of tumors. MiR-302e is a highly a ed typu RNA. $^{17}$ However, the role of miR-302e h lioma remains unclear. In present study, we firstly foun a disease of miR-302e in glioma clinical tissues and cell lines. Among glioma cell lines, miR-302e was lowest expressed in SHG44 cells, and highest expressed in U251 cells. Thus, we forced miR302e in SHG44 cells, and miR-302e promoted apoptosis and inhibited the growth, migration and invasion. Then, we silenced miR-302e in U251 cells, miR-302e inhibitor played the opposite function comparing with agomiR302e. These data suggested that miR-302e was involved in glioma development, and miR-302e might be a diagnose biomarker of glioma.

VEGFA, a vital gene in angiogenesis, can induce endothelial cell proliferation, promote cell migration. ${ }^{28,29}$ Some studies have found that VEGFA expression is increased in tumors, which is closely related to tumor staging and progression. ${ }^{30,31}$ Furthermore, VEGFA has been reported to be a target of miR-302e in ovarian cancer, and miR-302e inhibited VEGFA expression. ${ }^{19}$ In our study, we surprisedly found that there were paired bases between miR-302e and VEGFA. And further experiments revealed that miR-302e directly targeted VEGFA. Functional experiments showed that VEGFA removed the inhibitory effect of miR-302e on glioma progression. Furthermore, we constructed two in vivo tumor formation models to examine the role of miR-302e on glioma tumorigenesis. And we got the same conclusion, which was that miR-302e could significantly inhibit the glioma growth, which was conducive to clinical targeted therapy. In addition, because metastasis is an important part of tumor progression, we performed animal experiments to assess 

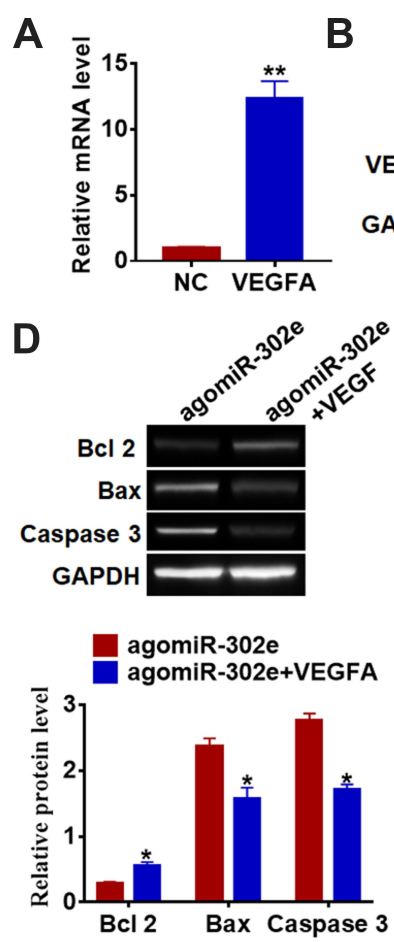

B

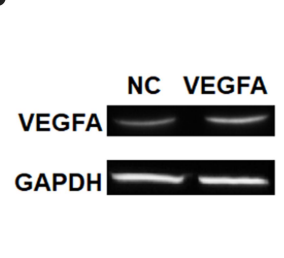

$\mathbf{E}$
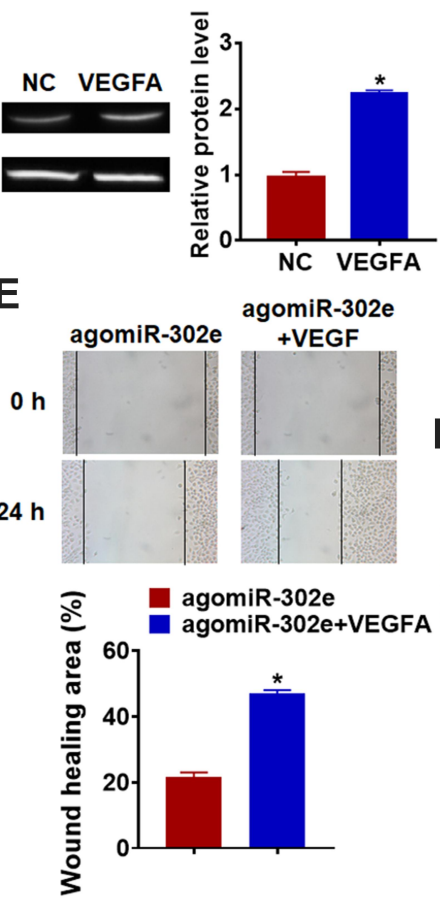

C
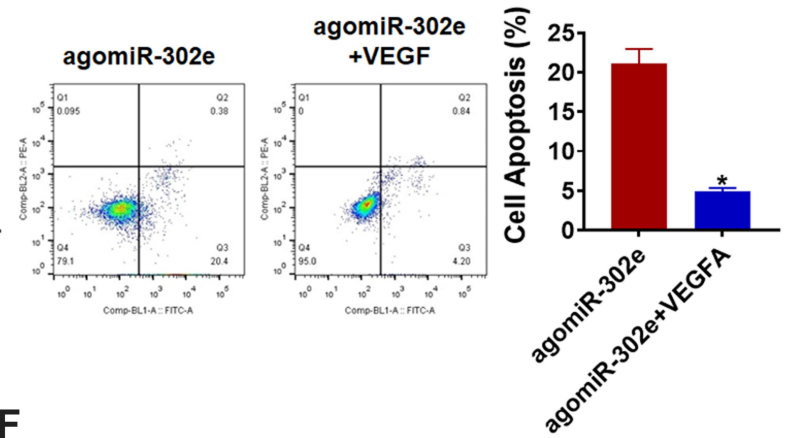

F
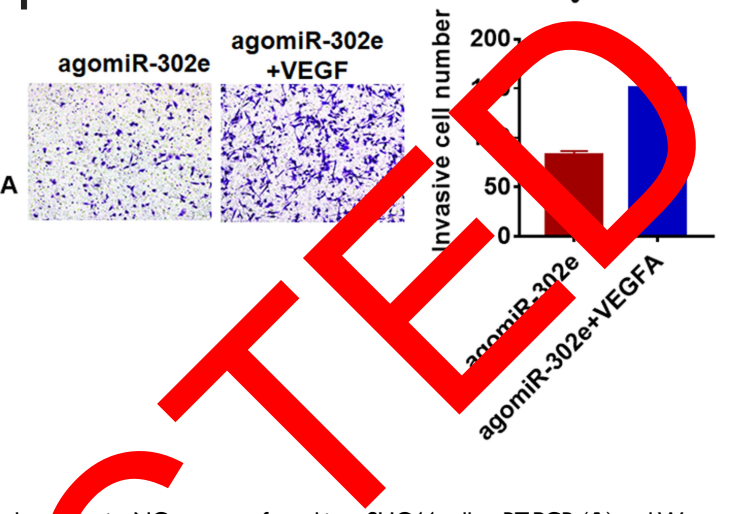
Figure 6 MiR-302e inhibits proliferation, migration and invasion by targeting VEGFA. VEGFA plasn or its NC was transfected into SHG44 cells. qRT-PCR (A) and Western blot (B)
was used to detect the transfection efficiency of VEGFA $\left({ }^{*} p<0.05\right.$, ${ }^{* *} p<0.01$ vs NC). (C) The apop sis of cells was ca ated by flow cytometry in SHG44 cells $\left({ }^{*} p<0.05\right.$ vs agomiR302e). (D) Western blot was performed to detected the expression of apoptosis related protein Bcl ax and caspase 3 ( $0.05 \mathrm{vs}$ agomiR-302e). (E) Wound healing assay was used to detect cell migration $(* p<0.05$ vs agomiR-302e). (F) Transwell assay was performed to check cell invasi times.

A

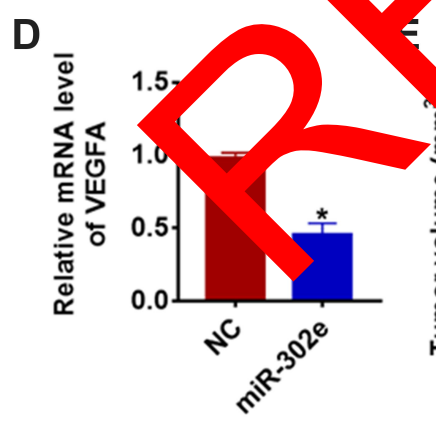

B
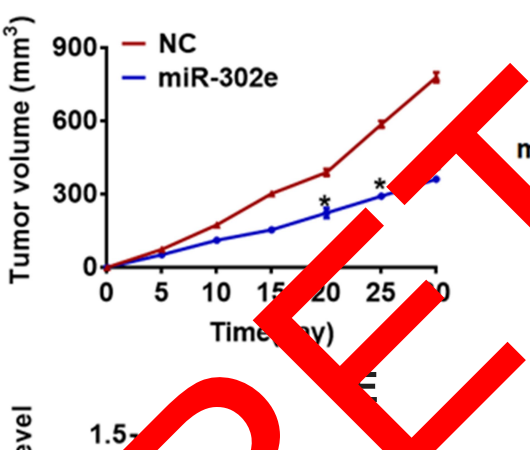
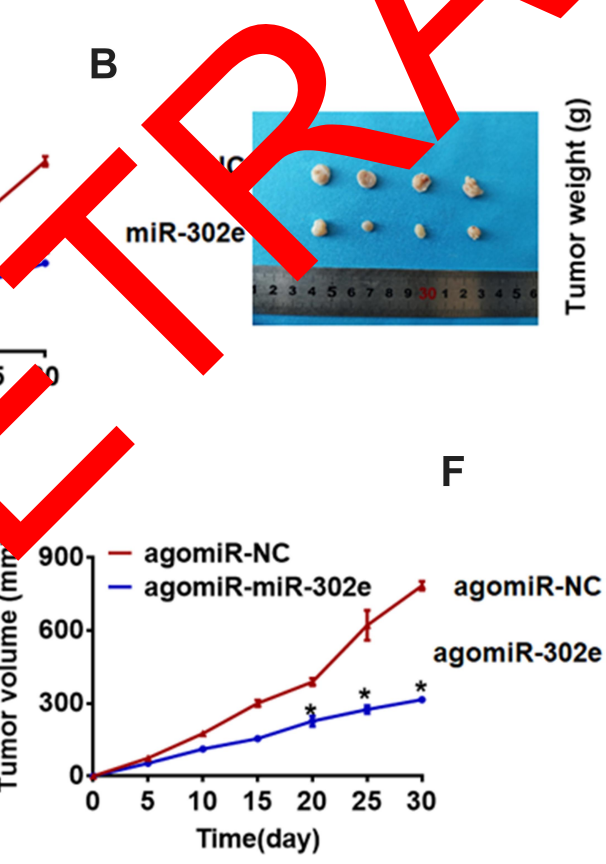

$\mathbf{F}$
C
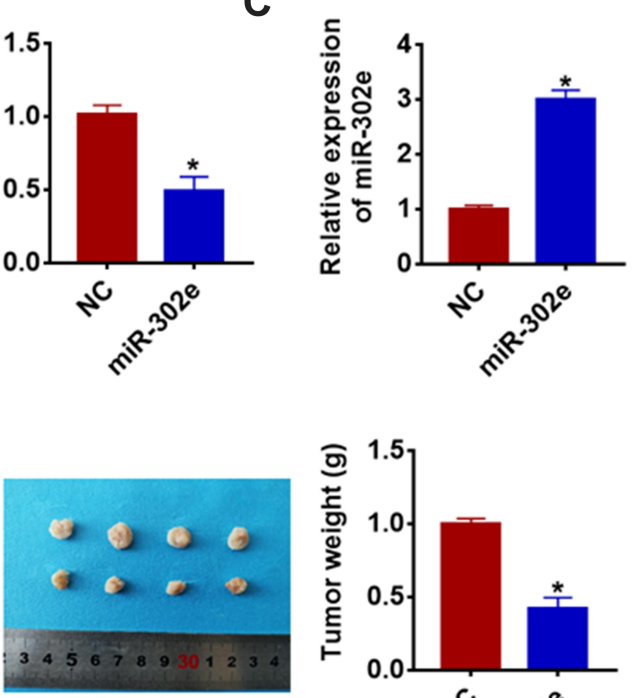

Figure 7 MiR-302e inhibits in vivo tumor growth in the nude mice. The nude mice were subcutaneously injected with SHG44 cells $\left(5 \times 10^{6}\right)$ stably transfected with miR$302 \mathrm{e}$ or NC in to the right flanks of the nude mice. (A) The tumor volume was assessed in the nude mice every 5 days $\left({ }^{*} p<0.05\right)$. (B) Tumor weight was determined in the isolated tumors from the nude mice $\left({ }^{*} p<0.05\right)$. (C) The relative expression of miR-302e was determined by qRT-PCR in the isolated tumor tissues $\left({ }^{*} p<0.05\right)$. (D) qRT-PCR was performed to detect the relative mRNA expression of VEGFA $\left({ }^{*} p<0.05\right)$. The nude mice were subcutaneously injected with SHG 44 cells $\left(5 \times 10^{6}\right)$, after 7 days of injection, miR-302e or its NC was injected into tumors. (E) The tumor volume was assessed in the nude mice every 5 days $\left({ }^{*} p<0.05\right)$. (F) Tumor weight was determined in the isolated tumors from the nude mice $\left({ }^{*} p<0.05\right)$. All experiments were carried repeatedly for 3 times. 
A

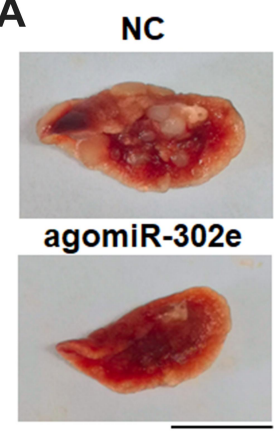

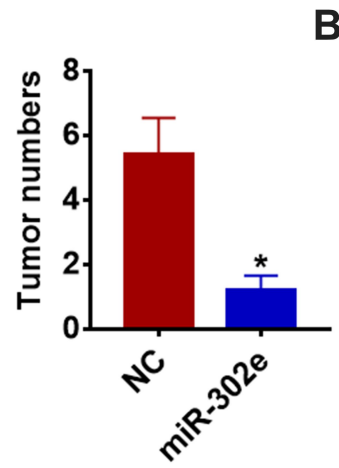
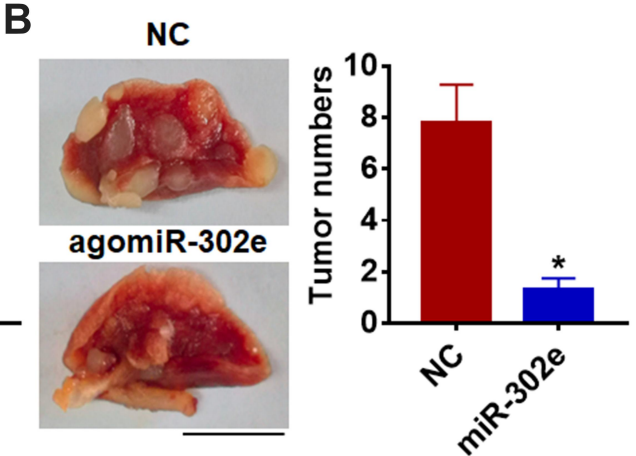

Figure 8 MiR-302e inhibits in vivo tumor metastasis in the nude mice. The nude mice were injected with SHG44 cells $\left(5 \times 10^{6}\right)$ sta ${ }^{2}$ ransin with miR-302e or NC through abdominal cavity $(\mathbf{A})$ and tail veins $(\mathbf{B})$, respectively. After 30 days of injection, the lungs were taken out and the tumors on lung were ca All experiments were carried repeatedly for 3 times $(* p<0.05)$.

pulmonary metastasis of glioma. We found that miR-302e could inhibit glioma metastasis, which may improve the prognosis of glioma patients.

Through understanding relevant studies on the involvement of miRNA in the regulation mechanism of glioma, the pathological processes such as proliferation, apoptosis, invasion and metastasis of glioma cells were deepened. At present, studies on the molecular level of glioma are still insufficient, so how to make miRNA play a better clinical application value in the diagnosis of glioma, evalua $f$ the efficacy of chemotherapy drugs, anti-angioge sis, treatment and prognosis judgment, etc. W/anther it provide a new way for the developmer of ca er trea ment drugs, these will be a long way go. It holieved that with the further study of $\mathrm{mi}^{\mathrm{P}} \mathrm{A}$ in cunction and mechanism of action of glio these pro ms will be gradually solved, which w brin new opponunities for clinical diagnosis and atment.

In conclusion, $r$ sent study revealce that miR-302e contributed to the rogre on of glioma, and miR-302e acted as an anti-tumo ene by geting VEGFA.

\section{Disc sur}

The author eclare no conflicts of interest for this work.

\section{References}

1. Haider AS, van den Bent M, Wen PY, et al. Towards a standard pathological and molecular characterization of recurrent glioma in adults: a RANO effort. Neuro Oncol. 2019.

2. Arnold A, Yuan M, Price A, Harris L, Eberhart CG, Raabe EH. Synergistic activity of mTORC1/2 kinase and MEK inhibitors suppresses pediatric low-grade glioma tumorigenicity and vascularity. Neuro Oncol. 2020;22(4):563-574.

3. Yekula A, Minciacchi VR, Morello M, et al. Large and small extracellular vesicles released by glioma cells in vitro and in vivo. $J$ Extracell Vesicles. 2020;9(1):1689784. doi:10.1080/20013078.2019.1689784
4. Han F, Zhang L, , et $\mathrm{GL}$. GR negatively regulates BRD4-dependent scriptio ongation a inhibits CRC metastasis. Adv Sci Vetm 9;6(23):1901114. doi:10.1002/advs.20 1901114

5. Han M, S, Fritah S, a nterfering with long non-coding RNA AIR2 $\mathrm{G}$ processing nhibits glioblastoma progression through suppro on of Wnt/beta-catenin signalling. Brain. $43(2): 512-3$

Xiong Y, Chen L, Ya. C, et al. Circulating exosomal miR-20b-5p inhibition tores wnt9b signaling and reverses diabetes-associated impaired wo hd healing. Small. 2019; e1904044.

Park K, Kiy KB. miRTar hunter: a prediction system for identifying roRNA target sites. Mol Cells. 2013;35(3):195-201. do1:10.1007/s10059-013-2165-4

-ang Q, Hu H, Yu X, et al. Tissue-specific microRNA expression aners cancer susceptibility conferred by a TP53 noncoding variant. Nat Commun. 2019;10(1):5061.

9. Huang H, Tang J, Zhang L, Bu Y, Zhang X. miR-874 regulates multiple-drug resistance in gastric cancer by targeting ATG16L1. Int J Oncol. 2018;53(6):2769-2779.

10. Sun W, Ping W, Tian Y, Zou W, Liu J, Zu Y. miR-202 enhances the anti-tumor effect of cisplatin on non-small cell lung cancer by targeting the Ras/MAPK pathway. Cell Physiol Biochem. 2018;51 (5):2160-2171. doi:10.1159/000495835

11. Li B, Wang W, Li Z, et al. MicroRNA-148a-3p enhances cisplatin cytotoxicity in gastric cancer through mitochondrial fission induction and cyto-protective autophagy suppression. Cancer Lett. 2017;410:212-227. doi:10.1016/j.canlet.2017.09.035

12. Xie C, Ji N, Tang Z, Li J, Chen Q. The role of extracellular vesicles from different origin in the microenvironment of head and neck cancers. Mol Cancer. 2019;18(1):83. doi:10.1186/s12943-019-0985-3

13. Xin Z, Jiang S, Jiang $\mathrm{P}$, et al. Melatonin as a treatment for gastrointestinal cancer: a review. J Pineal Res. 2015;58(4):375-387.

14. Selimoglu-Buet D, Riviere J, Ghamlouch H, et al. A miR-150/TET3 pathway regulates the generation of mouse and human non-classical monocyte subset. Nat Commun. 2018;9(1):5455. doi:10.1038/ s41467-018-07801-x

15. Ahn JH, Lee HS, Lee JS, et al. Author correction: nc886 is induced by TGF-beta and suppresses the microRNA pathway in ovarian cancer. Nat Commun. 2018;9(1):5458. doi:10.1038/s41467-01807818-2

16. Wu DM, Wang S, Wen X, et al. MircoRNA-1275 promotes proliferation, invasion and migration of glioma cells via SERPINE1. J Cell Mol Med. 2018;22(10):4963-4974. doi:10.1111/jcmm.13760

17. Chen $\mathrm{X}, \mathrm{Xu} \mathrm{Y}$, Liao $\mathrm{X}$, et al. Plasma miRNAs in predicting radiosensitivity in non-small cell lung cancer. Tumour Biol. 2016;37 (9):11927-11936. doi:10.1007/s13277-016-5052-8 
18. Cui D, Qian R, Li Y. Circular RNA circ-CMPK1 contributes to cell proliferation of non-small cell lung cancer by elevating cyclin D1 via sponging miR-302e. Mol Genet Genomic Med. 2020;8(2):e999.

19. Wang LL, Zong ZH, Liu Y, Guan X, Chen S, Zhao Y. CircRhoC promotes tumorigenicity and progression in ovarian cancer by functioning as a miR-302e sponge to positively regulate VEGFA. $J$ Cell Mol Med. 2019;23(12):8472-8481. doi:10.1111/jcmm.14736

20. Li D, Jiang X, Zhang X, Cao G, Wang D, Chen Z. Long noncoding RNA FGD5-AS1 promotes colorectal cancer cell proliferation, migration, and invasion through upregulating CDCA7 via sponging miR-302e. In Vitro Cell Dev Biol Anim. 2019;55(8):577-585. doi:10.1007/s11626-019-00376-x

21. Gritsenko PG, Atlasy N, Dieteren CEJ, et al. p120-catenin-dependent collective brain infiltration by glioma cell networks. Nat Cell Biol. 2020;22(1):97-107. doi:10.1038/s41556-019-0443-x

22. Jaraiz-Rodriguez $M$, Talaveron $R$, Garcia-Vicente $L$, et al. Connexin 43 peptide, TAT-Cx43266-283, selectively targets glioma cells, impairs malignant growth and enhances survival in mouse models in vivo. Neuro Oncol. 2020;22(4):493-504.

23. Li J, Zhang S, Wan Y, et al. MISIM v2.0: a web server for inferring microRNA functional similarity based on microRNA-disease associations. Nucleic Acids Res. 2019;47(W1):W536-W541. doi:10.1093/ nar/gkz328

24. Huang T, Wan X, Alvarez AA, et al. MIR93 (microRNA -93) regulates tumorigenicity and therapy response of glioblastoma by targeting autophagy. Autophagy. 2019;15(6):1100-1111. doi:10.10 80/15548627.2019.1569947
25. Wang R, Zhang S, Chen X, et al. EIF4A3-induced circular RNA MMP9 (circMMP9) acts as a sponge of miR-124 and promotes glioblastoma multiforme cell tumorigenesis. Mol Cancer. 2018;17 (1): 166 .

26. Ilisso CP, Delle Cave D, Mosca L, et al. S-Adenosylmethionine regulates apoptosis and autophagy in MCF-7 breast cancer cells through the modulation of specific microRNAs. Cancer Cell Int. 2018;18:197. doi:10.1186/s12935-018-0697-6

27. Shen G, Rong X, Zhao J, et al. MicroRNA-105 suppresses cell proliferation and inhibits PI3K/AKT signaling in human hepatocellular carcinoma. Carcinogenesis. 2014;35(12):2748-2755.

28. Thompson EM, Keir ST, Venkatraman T, et al. The role of angiogenesis in group 3 medulloblastoma pathogenesis and survival. Neuro Oncol. 2017;19(9):1217-1227. doi:10.1093/neuonc/nox033

29. Liu L, Bi N, Wu L, et al. MicroRNA- tions as a tumor suppressor by targeting VEGFA in ing adeno cinoma. Mol Cancer. 2017;16(1):50.

30. Wang H, Deng Q, Lv Z, et al. N6-meth enosine indu d miR-143$3 p$ promotes the brain metas is of lun ancer viz egulation of VASH1. Mol Cancer. 2019 (1):181

31. Teufel M, Seidel H, K ert K, a. Bioman s associated with response to regorafomib at with hematocellular carcinoma. Gastroenterology 19;156 $31-174$

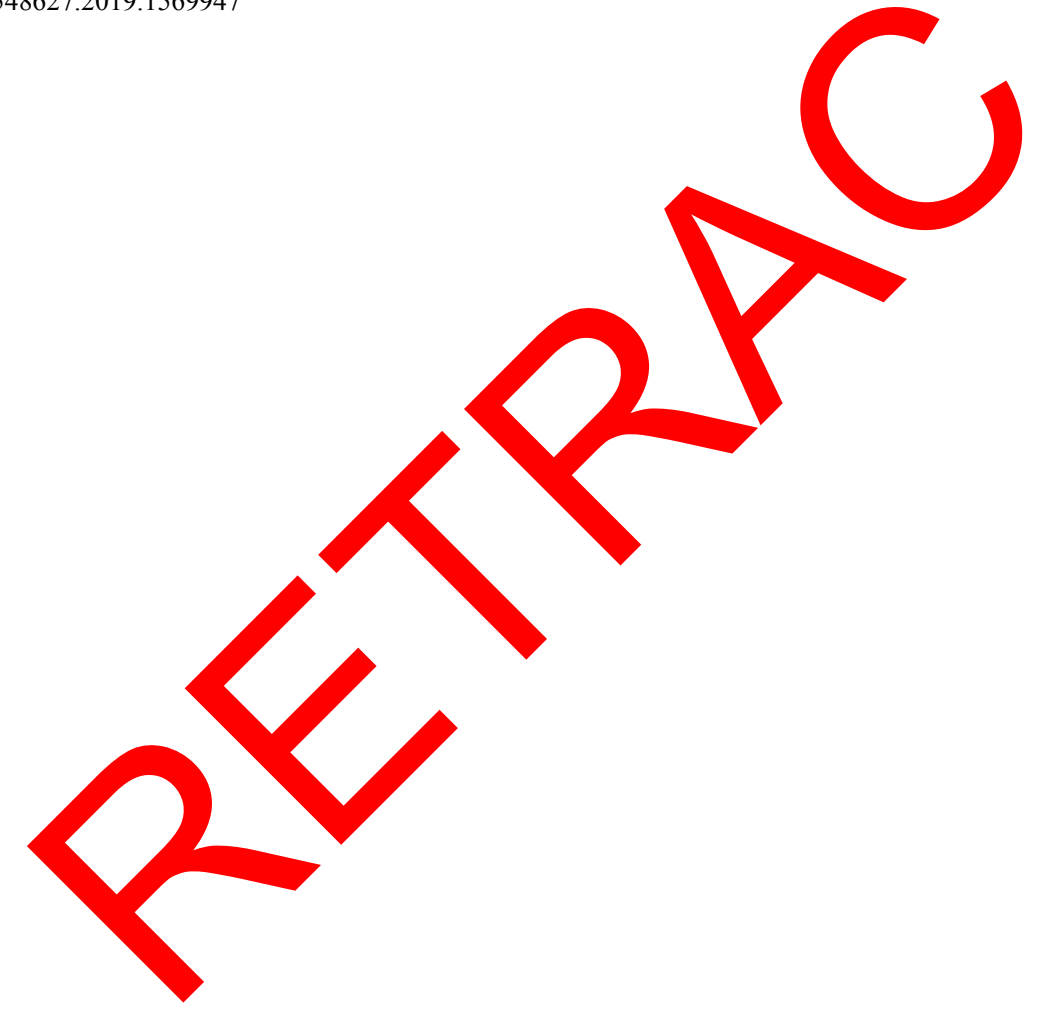

Cancer Management and Research

Dovepress

\section{Publish your work in this journal}

Cancer Management and Research is an international, peer-reviewed open access journal focusing on cancer research and the optimal use of preventative and integrated treatment interventions to achieve improved outcomes, enhanced survival and quality of life for the cancer patient.
The manuscript management system is completely online and includes a very quick and fair peer-review system, which is all easy to use. Visit http://www.dovepress.com/testimonials.php to read real quotes from published authors. 